Marquette University

e-Publications@Marquette

Psychology Faculty Research and Publications

Psychology, Department of

$12-1-2013$

\title{
Post-inpatient Attrition from Care "As Usual" in Veterans with Multiple Psychiatric Admissions
}

Nicholas W. Bowersox

Marquette University

Stephen M. Saunders

Marquette University, stephen.saunders@marquette.edu

Bertrand Berger

Clement J. Zablocki VA Hospital

Accepted version. Community Mental Health Journal, Vol. 49, No. 6 (December 2013): 694-703. DOI. (C) 2017 Springer Nature Switzerland AG. Part of Springer Nature. Used with permission. Sharable Link Provided by the Springer Nature SharedIt content-sharing initiative. 


\section{Marquette University}

\section{e-Publications@Marquette}

\section{Psychology Faculty Research and Publications/College of Arts and Sciences}

This paper is NOT THE PUBLISHED VERSION; but the author's final, peer-reviewed manuscript. The published version may be accessed by following the link in the citation below.

Community Mental Health Journal, Vol. 49, No. 6 (December, 2013): 694-703. DOI. This article is (C) Springer and permission has been granted for this version to appear in e-Publications@Marquette. Springer does not grant permission for this article to be further copied/distributed or hosted elsewhere without the express permission from Springer.

\section{Post-inpatient Attrition from Care "As Usual" in Veterans with Multiple Psychiatric Admissions}

Nicholas W. Bowersox

Serious Mental IIIness Treatment Research and Evaluation Center, Ann Arbor, MI

Stephen M. Saunders

Marquette University, Milwaukee, Wi

Bertrand Berger

Clement J. Zablocki VA, Hospital Milwaukee, WI

\section{Abstract}

Disengagement from outpatient care following psychiatric hospitalization is common in high-utilizing psychiatric patients and contributes to intensive care utilization. To investigate variables related to treatment attrition, a range of demographic, diagnostic, cognitive, social, and behavioral variables were collected from 233 veterans receiving inpatient psychiatric services who were then monitored over the following 2 years. During the follow-up period, $88.0 \%(n=202)$ of patients disengaged from postinpatient care. Attrition was associated with male gender, younger age, increased expectations of stigma, less short-term participation in group therapy, and poorer medication adherence. Of those who left care, earlier attrition was predicted by fewer prior-year inpatient psychiatric days, fewer lifetime 
psychiatric hospitalizations, increased perceived treatment support from family, and less short-term attendance at psychiatrist appointments. Survival analyses were used to analyze the rate of attrition of the entire sample as well as the sample split by short-term group therapy attendance. Implications are discussed.

\section{Keywords}

Veterans Treatment attrition; Psychiatric patients; High-utilizing patients; Survival analysis

\section{Introduction}

A subset of psychiatric patients accounts for a sizable proportion of emergency psychiatric service use. These patients are characterized by multiple inpatient psychiatric admissions, limited engagement in outpatient psychiatric care (Walker et al. 1996; Kent and Yellowlees 1994), and increased disability (Webb et al. 2007). Such patients constitute approximately 10$20 \%$ of psychiatric patients (Bobo et al. 2004; Roick et al. 2004; Vogel and Hugelet 1997) while accounting for a disproportionately large percentage of mental health costs.

There are somewhat inconsistent findings related to the level of post-inpatient care attrition of this group, in part due to inconsistencies in the definition and measurement of attrition (O'Brien et al. 2009). One review found that rates of attrition ranged from 18-67 \% across various studies, with a median rate of $58 \%$ (Kreyenbuhl et al. 2009). Additionally, a review of longterm treatment studies found that approximately $30 \%$ of psychiatric patients left mental health care over periods ranging from 1-9 years (O'Brien et al. 2009). Additional work appears necessary to clarify of how common care attrition is for psychiatric patients, but there is consensus that the rate is unacceptably high.

High rates of inpatient psychiatric service use of this population may be, in part, due to limited outpatient mental health treatment participation following discharge from inpatient psychiatric care. Outpatient treatments designed to address elevated psychiatric symptoms have been shown to be effective if patients are actively engaged (e.g., Lehman and Steinwachs 2003), and a lack of participation in outpatient mental health treatment has been linked to psychiatric readmission (Nelson et al. 2000; Prince 2005). This suggests the importance of keeping patients involved in treatment following discharge in order to reduce the "revolving-door" phenomena of repeated discharges and re-admissions to inpatient psychiatric care. Further supporting this idea, patient participation in mandatory outpatient psychiatric care has been demonstrated to result in reduced psychiatric admissions (Hunt et al. 2007; Rohland et al. 2000).

Although precise figures are difficult to establish, treatment attrition is a substantial concern with high utilizing psychiatric patients. Several variables have been implicated to play a role in treatment engagement following discharge from inpatient psychiatric care, including patient characteristics, patients' attitude towards treatment, social support, and patients' prior treatment experiences. 


\section{Demographic and Clinical Characteristics}

Research suggests that the psychiatric patients most likely to leave treatment prematurely tend to be younger, male, and members of racial/ethnic minority groups (Fischer et al. 2001; Kruse et al. 2002; McCarthy et al. 2007). Increased attrition has also been linked to the presence of comorbid substance use disorders and to more severe levels of psychiatric symptoms, although patients with psychotic disorders tend to have lower rates of treatment attrition relative to other diagnostic groups (Nose et al. 2003; O'Brien et al. 2009).

\section{Treatment Perspective}

There is evidence that patients' view of treatment influences their adherence to outpatient care. Attrition has been linked to dissatisfaction with treatment (Prince 2005), a desire to handle problems without outside assistance (Kessler et al. 2001; Priebe et al. 2005), and the perception that treatment is unnecessary (Kreyenbuhl et al. 2009; Rossi et al. 2008; Young et al. 2000). Patients also report a variety of barriers to care that interfere with their ability to actively participate in treatment, including the cost of care, time limitations, and concerns about stigma (Rossi et al. 2008; Vogt 2011; Young et al. 2000). Treatment attrition has likewise been linked to a poor working relationships with treatment professionals (O'Brien et al. 2009).

\section{Social Support}

Interpersonal relationships have been identified as playing important roles in supporting treatment engagement. Nonattendance at an intake session for outpatient care has been linked to limited family support (Kruse et al. 2002; Fischer et al. 2008a, $\underline{b}$ ). Conversely, a recent study found that the majority of psychiatric patients engaged in outpatient care experienced pressure to adhere to treatment from at least one person in the previous 6 months, with treatment professionals and family members representing the most common sources of treatment support (Redlich and Monahan 2006).

\section{Previous Treatment Engagement}

For some psychiatric patients, treatment engagement stops at the point of discharge from the inpatient psychiatric ward, with little or no participation in aftercare (Lingham and Scott 2002; Olfson et al. 2000). This initial post-discharge period appears to be the most likely time for patients to leave care (O'Brien et al. 2009), whereas initial participation in outpatient care is associated with treatment retention and more long-term treatment participation (Kreyenbuhl et al. 2009; Kruse et al. 2002).

\section{The Present Study}

The present study was designed to identify factors related to post-inpatient mental health treatment attrition in high-utilizing VA psychiatric patients. The study tracked patients over a period of 2 years following discharge from inpatient psychiatric care and utilized a well- 
validated measure of treatment participation. A broad range of variables thought to be related to engagement were measured and investigated to better understand patient attrition from care.

The present study had the following goals:

1. Evaluate the rate of post-inpatient treatment attrition of high-utilizing psychiatric patients over time.

2. Identify cognitive, social, and treatment variables associated with attrition from post-inpatient psychiatric care in high utilizing psychiatric patients.

3. Identify cognitive, social, and treatment variables associated with a more rapid departure from post-inpatient treatment in high utilizing psychiatric patients.

\section{Method}

\section{Treatment Perceptions Questionnaire}

Due to a lack of existing metrics to study treatment-related cognitions of psychiatric patients, the Treatment Perception Questionnaire (TPQ) was developed and utilized . First, focus groups were conducted with patients and providers within the inpatient psychiatric ward of a large VA hospital in the Midwest. Participants engaged in group-based conversations related to their expectations of the consequences of treatment participation and of persons who might play important roles in supporting or undermining treatment. Second, focus group information was used to create TPQ items that measured patients' expectations of factors associated with post-discharge care. The TPQ has two major scales, related to perceived consequences of treatment and perceived support for treatment. Each scale comprised subscales.

\section{Perceived Consequences Subscales}

Patients provided two responses to a list of 31 items that described potential consequences (i.e., their expectations of the ways that treatment participation would change their lives) of continuing treatment. First they rated the likelihood of each potential consequence, then they rated the potential tolerability of that consequence. Subscales were created to reflect the perceived consequences. The subscales were labeled Interpersonal Stigma, Intrapersonal Stigma, Material Cost of treatment, Changes in Relationships with others, and Changes in Symptom levels. Subscale scores ranged from -21.0 to 21.0, with lower scores representing increased levels of expected negative consequences as a result of treatment participation. Perceived consequences scales had fair to good internal consistency, with scale alphas between 0.60 and 0.80 .

\section{Perceived Social Support for Treatment Subscales}

Twelve TPQ items assessed the level of support for treatment that patients expected to receive from other people in their lives. Utilizing these items, subscales were created to 
represent the level of treatment support that patients expected from their Family Members and Treatment Providers. These TPQ subscales had subscale ranges from -21.0 to 21.0 , with higher scores representing increased perceived treatment support from that social support. Perceived Social Support subscales demonstrated fair to good internal consistency, with the Family Member subscale having a scale alpha of 0.65 and the Treatment Providers subscale having a scale alpha of 0.87 .

\section{Treatment Participation}

Short-Term Treatment Engagement

Three treatment activities of interest were selected for investigation based on conversations with patients and treatment staff: attendance at psychiatrist appointments, attendance at group therapy sessions, and medication utilization. Three variables were created to assess patients' treatment participation in these activities during the 2 months after discharge from inpatient psychiatric care. Utilizing an approach adopted in previous studies (e.g., Lingham and Scott 2002), medication utilization was assessed by comparing the number of total prescriptions to the number renewed in time to avoid a lapse in administration. Attendance at group therapy sessions and psychiatrist appointments were calculated as the ratio of attended appointments to overall scheduled appointments.

\section{Long-Term Treatment Participation}

Each veteran was tracked for 2 years post-discharge. Whether they attended their scheduled appointments during each week of the follow-up period was recorded based on a review of their electronic medical records (EMRs). Using a standard suggested by the Health Plan Employer Data and Information Set (HEDIS, Corrigan and Nielsen 1993), 30 days (5 weeks) was selected as the maximum acceptable time between treatment contacts for a patient to be considered continuously engaged in care. HEDIS guidelines have been previously utilized in studies of post-discharge follow-up of psychiatric inpatients (Druss et al. 2004) and in evaluations of VA mental health care quality (Harris et al. 2009). Based on these guidelines, each veteran was rated as either remaining in treatment or leaving care during the 2 year follow-up period. For veterans who left treatment, the amount of time between inpatient discharge and loss of treatment contact was calculated.

\section{Demographic and Clinical Variables}

Participants' age, race, gender, relationship status, and diagnosis were collected from EMRs. Each patient was categorized as having a diagnosis of a mood, psychotic, substance dependence, anxiety, or axis II disorder at the time of study entry. Additional treatment information collected from EMRs included: the number of inpatient psychiatric treatment days during the past year, number of lifetime psychiatric hospitalizations, and Global Assessment of Functioning (American Psychiatric Association 2000) score at intake into inpatient psychiatric care. 
To be included in this study, each patient had to meet the criteria established by Langdon et al. (2001) to be classified as a high-utilizer of services: (1) three or more lifetime psychiatric hospitalizations, (2) at least one psychiatric hospitalization in the year prior to the study, and (3) receiving inpatient psychiatric care at admission into the study. Each patient further was required to have prescribed psychiatric medications and planned post-discharge attendance at group therapy and psychiatric appointments as part of their post-inpatient psychiatric care.

A total of 314 veterans initially completed the questionnaire. Of those who completed the questionnaire, 65 were excluded either due to incomplete questionnaire completion, because they did not meet high-utilizer status, or because they did not have assigned participation in one of the treatment activities of interest within their discharge treatment plans. Another 16 patients were excluded because they belonged to groups with poor representation (e.g., 4 of Native American descent, 6 of Hispanic descent) and bivariate analyses suggested that they represented unique cohorts based on the relationship between predictor and outcome measures. This resulted in a final sample of 233 patients. This study was approved by the Institutional Review Board of the Veterans Affairs Healthcare Center at which the study was conducted.

\section{Procedure}

Patients receiving care within an inpatient psychiatric ward at a VA hospital in the Midwest were recruited between March and June of 2006 from within a combined inpatient treatment milieu setting wherein diagnostically diverse patients were treated in combined treatment groups and completed the TPQ in a group format. Participating patients completed an informed consent process and were offered $\$ 10$ in VA vouchers for their participation.

\section{Results}

Patient Characteristics

Patient demographic characteristics are presented in Table $\underline{1}$. Most patients were male and not in a committed relationship, and they were almost equally divided in terms of race. The sample was diagnostically diverse, and most had either or both a substance use and mood disorder. Patient clinical characteristics are also shown in Table $\underline{1}$, including GAF score when they entered the study, days of inpatient treatment over the year prior to the study, and lifetime total number of hospitalizations.

Table 1 Participant demographic, illness and treatment history variables $(N=233)$

Age

GAF score at intake into study Inpatient days in year prior to entry into study

Total hospitalizations-lifetime
$M$ (SD)

$8.76(9.67)$

$10.51(12.03)$ 


\begin{tabular}{|ll|}
\hline & \multicolumn{1}{c|}{$\boldsymbol{c}$ M (SD) } \\
Illness length (in years) & \multicolumn{1}{c|}{$\mathbf{N}(\%)$} \\
\hline \hline & \multicolumn{1}{c}{$\mathbf{N}(10.34)$} \\
Gender (male) & $218(93.6)$ \\
Race (African-American) & $111(47.6)$ \\
Relationship status (single) & $184(79.0)$ \\
Diagnosis & \\
Substance use disorder & $181(77.7)$ \\
Psychotic disorder & $34(14.6)$ \\
Mood disorder & $148(63.5)$ \\
Anxiety disorder & $56(24.0)$ \\
Personality disorder & $37(15.9)$ \\
\hline
\end{tabular}

a Diagnoses were given by VA mental health professionals. Diagnostic categories are not mutually exclusive. Substance Use Disorder includes alcohol, cocaine, and opioid dependence diagnoses. Psychotic Disorder includes diagnoses of schizophrenia, psychosis NOS, and bipolar disorder with psychotic features. Mood Disorder includes diagnoses of depression NOS, major depression, and bipolar disorder without psychotic features. Anxiety Disorder includes post-traumatic stress disorder, obsessive-compulsive disorder, and anxiety disorder NOS. Personality Disorder means diagnoses of personality disorders

\section{Prediction of Attrition from Care}

During the course of the 2-year follow-up period, 202 of 232 patients (88.0\%) dropped out of care based on a period of 5 weeks or greater without contact with their treatment staff. To evaluate attrition status, a logistic regression equation was calculated, with the dichotomous outcome variable of treatment engagement status at the end of the 2-year follow-up period (Table 2). Patient demographic characteristics, diagnosis, treatment history, and treatment expectation, as well as short-term treatment engagement measures, were used as predictor variables. This resulted in a significant regression model $\left(X^{2}(21)=55.11, p<.001, R^{2}=0.87\right)$. Attrition was significantly associated with younger age, male gender, increased expectations of intrapersonal stigma as a result of treatment, less short-term attendance at group therapy sessions, and fewer medications picked up from the pharmacy in the 3 months following discharge from inpatient care.

Table 2 Summary of logistic regression analyses predicting treatment attrition status $(N=233)$

\begin{tabular}{|lllll|}
\hline \multicolumn{1}{|c}{ Predictor variable } & \multicolumn{1}{c}{ B } & SE $\boldsymbol{B}$ & \multicolumn{1}{c}{ OR } & $\mathbf{9 5} \% \mathbf{C l}$ for OR \\
Demographic measures & & & & \\
Age & $0.09^{*}$ & 0.04 & 1.10 & $1.01-1.19$ \\
Gender (1 = male) & $2.43^{\star *}$ & 0.96 & 10.41 & $1.57-68.90$ \\
Race (1 = African-American) & 0.27 & 0.53 & 1.31 & $0.47-3.68$ \\
Treatment measures & & & & \\
GAF score at admission & -0.04 & 0.03 & 0.96 & $0.91-1.01$
\end{tabular}




\begin{tabular}{|lllll|}
\hline \multicolumn{1}{|c}{ Predictor variable } & B & \multicolumn{1}{c|}{ SE B } & \multicolumn{1}{c|}{ OR } & \multicolumn{1}{c|}{$\mathbf{9 5}$ \% CI for OR } \\
Inpatient days in year prior & 0.02 & 0.02 & 1.02 & $0.98-1.07$ \\
Number of lifetime inpatient admissions & 0.03 & 0.02 & 1.03 & $0.99-1.07$ \\
Diagnostic status (1 = positive) & & & & \\
Substance use disorder & 0.56 & 0.61 & 1.74 & $0.53-5.74$ \\
Psychotic disorder & 0.11 & 0.80 & 1.12 & $0.23-5.34$ \\
Mood disorder & 0.81 & 0.62 & 2.24 & $0.67-7.51$ \\
Anxiety disorder & -0.44 & 0.60 & 0.64 & $0.20-2.08$ \\
Personality disorder & -0.93 & 0.61 & 0.39 & $0.12-1.29$ \\
Treatment expectations & & & & \\
Expected social stigma from treatment & 0.06 & 0.05 & 1.06 & $0.97-1.17$ \\
Expected internal stigma from treatment & -0.14 & 0.07 & $0.87^{\star}$ & $0.76-0.99$ \\
Expected material cost of treatment & -0.08 & 0.08 & 0.92 & $0.79-1.08$ \\
Expected social consequences of treatment & -0.01 & 0.07 & 1.00 & $0.88-1.14$ \\
Expected symptom change from treatment & 0.06 & 0.06 & 1.06 & $0.94-1.19$ \\
Perceived treatment support from family & -0.04 & 0.04 & 0.96 & $0.88-1.04$ \\
Perceived treatment support from treatment staff & 0.03 & 0.05 & 1.03 & $0.94-1.13$ \\
Short-term treatment engagement measures & & & & \\
Psychiatrist appointment attendance & 0.22 & 0.61 & 1.25 & $0.38-4.13$ \\
Group therapy attendance & 2.50 & 0.93 & $12.18^{* *}$ & $1.97-75.34$ \\
Medication pick-up & 1.60 & 0.75 & $4.71^{*}$ & $1.08-20.54$ \\
\hline
\end{tabular}

Model: $\chi 2(21)=55.11, p<.001$, Adjusted $R 2=.87$

${ }^{\star} p<.05 ;{ }^{* *} p<.01 ;{ }^{* \star *} p<.001$

A second regression equation was calculated to predict time until treatment attrition, using only those veterans who left treatment during the follow-up period $(n=202)$. Once again, patient demographic, diagnostic, treatment history, treatment expectation, and short-term treatment engagement measures were used as predictor variables. The results are summarized in Table 3. The resultant model was significant $(R=0.54, F(21,180)=3.50, p<.001$, $R 2=0.29)$. Earlier attrition was significantly associated with fewer inpatient psychiatric days in the year prior to the study, fewer lifetime psychiatric hospitalizations, increased expectations of material cost resulting from treatment participation, increased perceived treatment support from family members, less short-term post-discharge attendance at psychiatrist appointments, and less short-term post-discharge attendance at group therapy sessions.

Table 3 Summary of regression analyses predicting time to treatment attrition $(N=202)$

Predictor variable

Demographic measures

Age

\section{B SE B t}

$\begin{array}{lll}0.22 & 0.19 & 1.18\end{array}$




\begin{tabular}{|llll|}
\hline \multicolumn{1}{|c}{ Predictor variable } & B & SE & \multicolumn{1}{c|}{$\boldsymbol{t}$} \\
Gender (1 = male) & 5.36 & 5.87 & 0.91 \\
Race (1 = African-American) & 2.35 & 2.92 & 0.81 \\
Treatment measures & & & \\
GAF score at admission & 0.26 & 0.15 & 1.70 \\
Inpatient days in year prior & 0.73 & 0.18 & $4.03^{\star \star}$ \\
Number of lifetime inpatient admissions & 0.33 & 0.14 & $2.40^{\star}$ \\
Diagnostic status (1 = positive) & & & \\
Substance use disorder & 6.22 & 3.71 & 1.68 \\
Psychotic disorder & -2.32 & 4.44 & -0.52 \\
Mood disorder & 2.91 & 3.17 & 0.92 \\
Anxiety disorder & 5.14 & 3.55 & 1.45 \\
Personality disorder & 3.48 & 4.01 & 0.87 \\
Treatment expectations & & & \\
Expected social stigma from treatment & -0.06 & 0.25 & -0.26 \\
Expected internal stigma from treatment & -0.15 & 0.40 & -0.39 \\
Expected material cost of treatment & 0.89 & 0.41 & $2.18^{\star}$ \\
Expected social consequences of treatment & -0.22 & 0.41 & -0.55 \\
Expected symptom change from treatment & 0.22 & 0.32 & 0.71 \\
Perceived treatment support from family & -0.51 & 0.25 & $-1.99^{\star}$ \\
Perceived treatment support from treatment staff & 0.15 & 0.24 & 0.62 \\
Short-term treatment engagement measures & & & \\
Psychiatrist appointment attendance & 8.76 & 3.36 & $2.61^{\star \star}$ \\
Group therapy attendance & 8.36 & 3.70 & $2.26^{\star}$ \\
Medication pick-up & 3.19 & 3.91 & 0.82 \\
\hline
\end{tabular}

Model: $F(21,180)=3.50, p<.001$, Adjusted $R 2=.29$

${ }^{\star} p<.05 ;{ }^{* \star} p<.01 ;{ }^{* \star \star} p<.001$

$\mathrm{S}$

urvival Analyses of Treatment Attrition Status

Survival analyses were conducted to predict treatment attrition for the entire sample of 233 patients, and the associated survival plot is displayed in Fig. 1 . The results illustrated a high initial loss of veterans followed by a steady loss of additional veterans throughout the follow-up period. Several points of interest were identified to better describe this graph in Table $\underline{4}$. Note that discrepancies between numbers and percentages reflect adjustments for loss of veterans who did not complete the full follow-up period (due to death or delayed release from intensive care). 


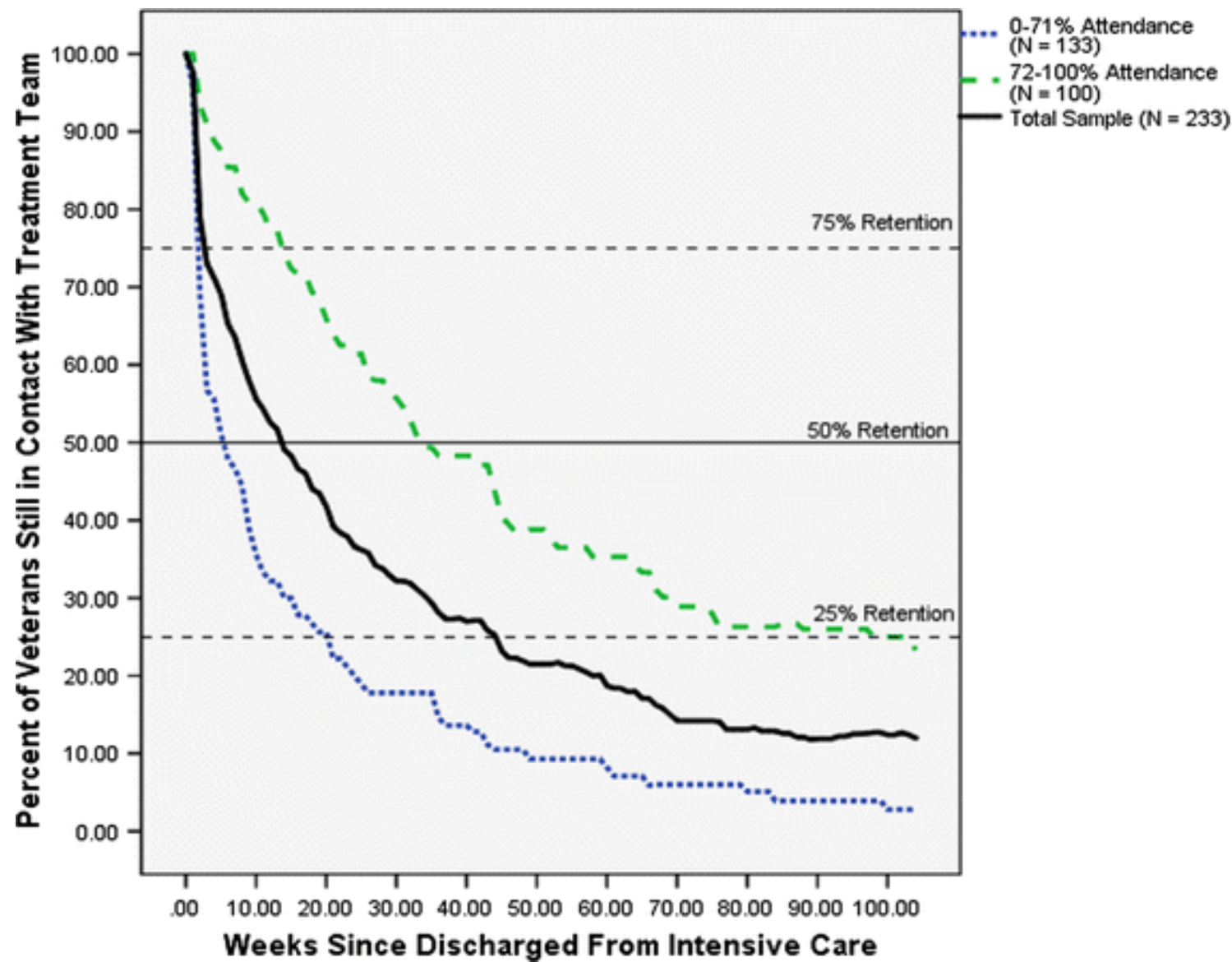

Fig. 1 Treatment contact split by short-team group attendance $(N=233)$

Table 4 Treatment contact survival analysis results: total sample and sample split by short-term postdischarge attendance at group therapy sessions $(N=233)$

\begin{tabular}{|c|c|c|c|c|c|c|c|c|c|c|}
\hline \multirow[t]{2}{*}{ Survival } & \multicolumn{3}{|c|}{$\begin{array}{c}\% \quad \text { Total sample } \\
(N=233)\end{array}$} & \multicolumn{3}{|c|}{$\begin{array}{l}\text { Low short-term group } \\
\text { treatment attendance } \\
\qquad(N=133)\end{array}$} & \multicolumn{4}{|c|}{$\begin{array}{l}\text { High short-term group } \\
\text { treatment attendance } \\
\qquad(N=100)\end{array}$} \\
\hline & 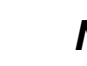 & \multicolumn{2}{|c|}{ Week Slope $^{a}$} & \multicolumn{2}{|r|}{ Week } & Slope & \multicolumn{3}{|c|}{ Week } & Slope \\
\hline 100 & & 30 & $n / a^{b}$ & 133 & 0 & $\mathrm{n} / \mathrm{a}$ & 100 & 0 & & $\mathrm{n} / \mathrm{a}$ \\
\hline 75 & & 63 & -19.40 & 95 & 3 & -8.31 & 73 & 9 & & -5.00 \\
\hline 50 & & 514 & -5.29 & 65 & 11 & -3.62 & 49 & 28 & & -1.79 \\
\hline 25 & & 44 & -1.93 & 32 & 33 & -1.52 & 24 & 77 & & -0.67 \\
\hline \multirow[t]{2}{*}{ Final } & 2 & 104 & -0.51 & 6 & 104 & -0.33 & 15 & 104 & & -0.17 \\
\hline & \multicolumn{2}{|c|}{ Final \% } & \multicolumn{2}{|c|}{ Overall slope } & Final \% & \multicolumn{2}{|c|}{ Overall slope } & Final \% & \multicolumn{2}{|c|}{ Overall slope } \\
\hline Overall & 12. & & 1.98 & & 7.00 & -1.18 & & 18.50 & -0.78 & \\
\hline \multicolumn{4}{|c|}{ Variable } & \multicolumn{3}{|c|}{$\begin{array}{l}\text { Low attendance } \\
\qquad(N=133)\end{array}$} & $\begin{array}{l}\mathrm{h} \text { atte } \\
(N=:\end{array}$ & $\begin{array}{l}\text { endance } \\
100)\end{array}$ & & $\overline{x^{2}}$ \\
\hline \multicolumn{4}{|c|}{ In treatment after 2 years } & 7.50 & $\%$ & 18.5 & & & $1-$ & $-2.14^{*}$ \\
\hline
\end{tabular}




\begin{tabular}{|crrrrrc|}
\hline Variable & $\boldsymbol{M}$ & SD & $\boldsymbol{M}$ & SD & $\boldsymbol{d f}$ & $\mathbf{X}^{\mathbf{2}}$ \\
Time to treatment attrition & 15.63 & 19.25 & 22.26 & 23.35 & 156.76 & $7.46^{\star \star}$ \\
\hline
\end{tabular}

${ }^{*} p<.05 ;{ }^{* *} p<.01$

a Slope measured in average number of patients lost to treatment contact for 5 weeks or longer per week for the period between that and the previous point in the survival plot

${ }^{b}$ Slopes are not listed for $100 \%$ survival points as these represent the beginning points of the survival plots, with no previous points on the line with which to generate a slope

\section{Post-Hoc Analyses Using Short-Term Group Attendance}

Upon reviewing the results of the regression analyses, it appeared that post-discharge shortterm group therapy attendance was an important factor in both treatment attrition and time to attrition. Post-hoc analyses were conducted to examine this variable further. First, two groups were created based on a median split on group therapy attendance $(M=0.71)$. One group (Low Attendance) comprised patients with $71 \%$ or less rate of group attendance $(N=133)$ and the other group (High Attendance) comprised those with $72 \%$ or more rate of attendance $(N=100)$. Next, new survival plots were generated for each of these two groups, and these results are also presented in Fig. 1 . Results indicated that $18.5 \%$ of the High Attendance group members survived until the end of the follow-up period, compared to $7.00 \%$ of the Low Attendance group members $(\chi 2[\mathrm{df}=1]=2.14, p<.05)$. For the High Attendance group members, the average time to attrition (in days) was $22.26(S D=23.35)$ versus $15.63(S D=19.25)$ for the Low Attendance group $(\mathrm{t}=7.46, \mathrm{p}<.001)$.

\section{Discussion}

The problem of psychiatric patients utilizing unusually high levels of resources, especially expensive inpatient care, is paradoxically related to limited participation in outpatient care (e.g., Prince 2005). In this study, we attempted to evaluate further person-specific factors related to poor compliance with outpatient follow-up care by patients recently discharged from inpatient units.

Across the entire sample, $88.0 \%$ left treatment during the 2-year follow-up period, with $67.80 \%$ attrition by 6 months, $81.60 \%$ attrition at 1 year, and $87.40 \%$ attrition by 18 months. These numbers are noticeably higher than the $30 \%$ rate reported by O'Brien et al. (2009) or the $58 \%$ rate cited by Kreyenbuhl et al. (2009). These discrepancies in attrition rates suggest that further research into the rates of longitudinal attrition in psychiatric patients is merited and point to the need for standardized definitions and measures of attrition. The use of a standardized measure of treatment adequacy within this study (the HEDIS measure of 5 weeks between treatment contacts) represents a positive step toward the establishment of such definitions and measurement metrics.

These results suggest that younger male patients were most likely to leave treatment, especially if they expected to experience more internal stigma as a result of treatment. These results also illuminated some early indicators of potential attrition. Patients who were less compliant with medication prescriptions (i.e., who picked up their prescriptions from the 
pharmacy less frequently) and who had poorer short-term attendance at post-discharge group therapy sessions were most likely to disengage from ongoing outpatient treatment and to disengage more quickly.

These findings are similar to previous work in this area. Younger age and male gender have been found to be associated with a loss of treatment contact within VA psychiatric patients (McCarthy et al. 2007; O'Brien et al. 2009). Likewise, concerns related to negative self-image have been established as barriers to care engagement (e.g., Perese 2007). As far as we know, this is the first study to show a relationship between treatment attrition and poor participation in specific treatment behaviors (i.e., group and medications) in the immediate period following discharge from inpatient care.

Among patients who left treatment, earlier departure was associated with fewer prior-year inpatient psychiatric days, fewer lifetime psychiatric hospitalizations, increased expectations of material cost of treatment, higher levels of perceived family treatment support, and less shortterm post-discharge attendance at psychiatric and group therapy session appointments The association between past and present service utilization may reflect a lack of perceived need for treatment by the patients in the study, a factor important in supporting treatment engagement (Elbogen et al. 2006). While the relationship between short-term and long-term engagement may be due in part to an overlap in the measurement of these variables, it is interesting to note that the treatment behaviors associated with care retention all had a social interaction component, while the behavior unrelated to time to attrition (medication pickup) is performed in isolation.

The importance of short-term group therapy attendance is worth further exploration. Almost the entire group (97.2\%) of the low attendance group members left during the 2-year follow-up period, while only $75 \%$ of the high attendance group left care. There were also differences in attrition rates between the two groups, with the low attendance group reaching the $25 \%$ attrition mark seven times more quickly and both the 50 and $75 \%$ attrition mark more than five times as quickly as the high attendance group. These groups had different patterns of attrition, with the high attendance group leaving in a steady manner throughout the follow-up period while the low attendance group experienced initial extreme attrition followed by steady, moderate-level loss.

Our study found that increased perceived family treatment support was associated with shorter time to attrition, a result that runs contrary to previous research on treatment engagement in psychiatric patients (e.g., Fischer et al. 2008a, b) and suggests the need for collaboration between families and caregivers of patients. The relationship between increased perceived cost of care and earlier attrition mirrors previous work that found increased attrition in patients who expected care to be more expensive or require increased time commitments (Rossi et al. 2002; Young et al. 2000). This suggests that patients transitioning from inpatient to outpatient care would benefit from problem-solving and case management services to assist them in managing resources necessary for effective participation in outpatient mental health care.

Also of note was the lack of significant relationships between variables that might be expected to predict attrition. Diagnosis was generally unrelated to treatment engagement, despite 
previous work that found that the presence of psychotic (Rossi et al. 2002), substance dependent (Ball et al. 2006; Fischer et al. 2001), and mood symptoms (Webb et al. 2007) are associated with differential rates of treatment engagement.

\section{Evidence for Subpopulations of Patients}

Overall, the results of the survival analyses suggest that there are multiple sub-populations of psychiatric patients, rather than the two ("engaged" versus "disengaged") considered in this study. The rates of attrition in the study suggest at least three groups of patients: those who leave treatment shortly after discharge from inpatient care, those who remain engaged throughout, and those who have initial care participation followed by later disengagement.

Evidence for a completely nonparticipatory sub-group can be seen in the drastic loss of patients following discharge from intensive psychiatric hospitalization, with more than $25 \%$ leaving within the first 3 weeks of care. A group committed to long-term engagement is suggested by the cohort remaining throughout the follow-up period $(12 \%$ of the overall sample). The remaining $63 \%$ of the graph does not clearly fit into either other group and is characterized by a gradually-decreasing slope with initially high levels of attrition that become more moderate over time. Differences in engagement of these three groups suggests differences in treatment perspective, with those immediately disengaging perhaps viewing treatment as unnecessary, those who are relatively committed perhaps seeing treatment as essential, and the rest viewing treatment as initially important but less so as symptoms stabilize. It is likely that increased patient functioning is associated with less perceived need for ongoing assistance in managing symptoms, a finding that is supported by previous research on reasons for treatment disengagement (Kreyenbuhl et al. 2009). The additional finding that the number of past-year psychiatric hospitalizations and lifetime hospitalizations were associated with treatment retention suggests that symptom chronicity and symptom severity may both shape the level of perceived need for treatment participation.

Supporting these ideas, the middle graph resembles the dose-response model (Howard et al. 1986), which suggests that patients terminate outpatient psychotherapy after an improvement in symptom levels. Within the dose-response curve, the rates of patients who had not yet experienced symptom improvement were: $64 \%$ after 3 sessions, $52 \%$ after 7 sessions, $32 \%$ after 16 sessions, $26 \%$ after 52 sessions, and $15 \%$ after 104 sessions (Howard et al. 1986). Within the present study, $71 \%$ of veterans remained in treatment after 3 weeks, $56 \%$ remained after 7 weeks, $46 \%$ remained after 16 weeks, $22 \%$ remained after 52 weeks, and $12 \%$ remained after 104 weeks. The similarity between these graphs suggests that persons within this third group may have experienced symptom reduction then chose to leave treatment as they felt that ongoing treatment participation was unnecessary for effective management of their symptoms, a pattern consistent with many other psychological conditions as well as previous research on psychiatric patients (O'Brien et al. 2009). Unfortunately, given the high level of both recent and past emergency psychiatric care utilization of this population, these results suggest that such patients may be overestimating their ability to independently manage their symptoms or may not realize the need for help until their symptoms are at emergent levels. Counter intuitively, providers might find better outcomes for high-utilizing patients by 
actively reaching out to patients who appear to require the least support following inpatient care.

A notable result of this study is the high rate of treatment attrition within high-utilizing psychiatric patients. Elevated rates of attrition occurred even within the group with the highest rate of treatment success, suggesting that such outcomes are characteristic of this population and likely to occur even when patients are provided with the most appropriate and wellimplemented treatment. The transition from inpatient to outpatient care appears to be a crucial period during which patients are very likely to leave. However, the increased retention associated with group therapy participation speaks to the importance of patient-to-patient relationships for psychiatric patients. These patients would likely benefit from staff efforts aimed at fostering such relationships, both during and immediately after inpatient psychiatric care.

\section{Study Limitations}

Several limitations affected study results. While the pool was well-distributed among some variables such as relationship status, age, and race, several other variables such as gender were highly constricted within the sample. Also of concern is the broad definition of treatment as "any contact between providers and patients." This measure likely inflated the estimate of treatment involvement, as persons were considered to be engaged even if they were only attending to portions of their prescribed care.

\section{Conclusions and Future Directions}

This study suggests that there are multiple sub-sets of high-utilizing psychiatric patients with regard to treatment engagement. It is likely that the process of treatment engagement is fluid, with patients entering and leaving treatment repeatedly over time rather than simply remaining in care throughout. While traditional survival analysis techniques allowed a partial investigation into the relationships between these variables, future work using repeated event survival analysis could provide insights into the way these variables play out in care.

These results also indicate that social and cognitive factors play important roles in preventing attrition from care for this group. The role of other important factors such as emotion or motivation have not been adequately investigated and hold the potential to allow a more complete understanding of the reasons for patient disengagement from outpatient mental health care.

This study represents a systematic evaluation of factors predictive of attrition from outpatient care by high-utilizing psychiatric patients through the use of a well-supported measure of treatment contact adequacy. It also represents a first step toward the development of interventions aimed at reducing emergency psychiatric services use by high-utilizing patients by highlighting clinical and treatment-related factors associated with attrition, assisting clinicians in identifying patients most likely to leave care and treatment factors (group therapy) associated with greater treatment retention for this group. 


\section{References}

1 American Psychiatric Association. (2000). Diagnostic and statistical manual of mental disorders (4th edn, text revision). Washington, DC: American Psychiatric Association.

2 Ball, S., Carroll, K., Canning-Bell, M., \& Rounsaville, B. J. (2006). Reasons for dropout from drug abuse treatment: Symptoms, personality, and motivation. Addictive Behaviors, 31(2), 320-330.

3 Bobo, W. V., Hoge, C. W., Messina, M. A., Pavolovic, F., Levandowski, D., \& Grieger, T. (2004). Characteristics of repeat users of an inpatient psychiatry service at a large military tertiary care hospital. Military Medicine, 169(8), 648-653.

4 Corrigan, J. M., \& Nielsen, C. M. (1993). Toward the development of uniform reporting standards for managed care organizations: The health plan employer data and information set (version 2.0). The Joint Commission on the Accreditation of Healthcare Organizations 19(12), 566-575.

5 Druss, B. G., Miller, C. L., Pincus, H. A., \& Shih, S. (2004). The volume-quality relationship of mental health care: Does practice make perfect? The American Journal of Psychiatry, 161(12), 2282-2286.

6 Elbogen, E. B., Van Dorn, R. A., Swanson, J. W., Swartz, M. S., \& Monahan, J. (2006). Treatment engagement and violence risk in mental disorders. British Journal of Psychiatry, 189, 354-360.

7 Fischer, E. H., Dornelas, E. A., \& Goethe, J. W. (2001). Characteristics of people lost to attrition in psychiatric follow-up studies. The Journal of Nervous and Mental Disease, 189(1), 49-55.

8 Fischer, E. P., McCarthy, J. M., Ignacio, R. V., Blow, F. C., Barry, K. L., Hudson, T. J., et al. (2008a). Longitudinal patterns of health systems retention among veterans with schizophrenia or bipolar disorder. Community Mental Health Journal, 44(5), 321-330.

9 Fischer, E. P., McSweeney, J. C., Pyne, J. M., Williams, D. K., Naylor, A. J., Blow, F. C., et al. (2008b). Influence of family involvement and substance use on sustained utilization of services for schizophrenia. Psychiatric Services, 59(8), 902-908.

10 Harris, A. H. S., Bowe, T., Finney, J. W., \& Humphreys, K. (2009). HEDIS initiation and engagement quality measures of substance use disorder care: Impact of setting and health care specialty. Population Health Management, 12(4), 191-196.

11 Howard, K. I., Kopta, S. M., Krause, M. S., \& Orlinsky, D. E. (1986). The dose-effect relationship in psychotherapy. American Psychologist, 41(2), 159-164.

12 Hunt, A. M., da Silva, A., Lurie, S., \& Goldbloom, D. S. (2007). Community treatment orders in Toronto: The emerging data. The Canadian Journal of Psychiatry, 52(10), 647-656.

13 Kent, S., \& Yellowlees, P. (1994). Psychiatric and social reasons for frequent rehospitalization. Hospital \& Community Psychiatry, 45(4), 347-350.

14 Kessler, R. C., Berglund, P. A., Bruce, M. L., Koch, J. R., Laska, E. M., Leaf, P. J., et al. (2001). The prevalence and correlates of untreated serious mental illness. Health Services Research, 36(6 pt 1), 987-1000.

15 Kreyenbuhl, J., Nossel, H. R., \& Dixon, L. B. (2009). Disengagement from mental health treatment among individuals with schizophrenia and strategies for facilitating connections to care: A review of the literature. Schizophrenia Bulletin, 35(4), 696-703.

16 Kruse, G. R., Rohland, B. M., \& Wu, X. (2002). Factors associated with missed first appointment at a psychiatric clinic. Psychiatric Services, 53(9), 1173-1176. 
17 Langdon, P. E., Yaguez, L., Brown, J., \& Hope, A. (2001). Who walks through the 'revolving door' of a British psychiatric hospital? Journal of Mental Health, 10(5), 525-533.

18 Lehman, A. F., \& Steinwachs, D. M. (2003). Evidence-based psychosocial treatment practices in schizophrenia: Lessons from the Patient Outcomes Research Team (PORT) project. Journal of the American Academy of Psychoanalysis and Dynamic Psychiatry, 31(1), 141-154.

19 Lingham, R., \& Scott, J. (2002). Treatment non-adherence in affective disorders. Acta Psychiatrica Scandinavica, 105(3), 164-172.

20 McCarthy, J. F., Blow, F. C., Valenstein, M., Fischer, E. P., Owen, R. R., Barry, K. L., et al. (2007). Veteran Affairs Health System and mental health treatment retention among patients with serious mental illness: Evaluating accessibility and availability barriers. Health Research and Educational Trust, 42(3 pt 1), 1042-1060.

21 Nelson, E. A., Maruish, M. E., \& Axler, J. L. (2000). Effects of discharge planning and compliance with outpatient appointments on readmission rates. Psychiatric Services, 51(7), 885-889.

22 Nose, M., Barbui, C., \& Tansella, M. (2003). How often do patients with psychosis fail to adhere to treatment programmes? A systematic review. Psychological Medicine, 157(7), 1149-1160.

23 O'Brien, A., Fahmy, R., \& Singh, S. P. (2009). Disengagement from mental health services. Social Psychiatry and Psychiatric Epidemiology, 44(7), 558-568.

24 Olfson, M., Mechanic, D., Hansell, S., Boyer, C. A., Walkup, J., \& Weiden, P. J. (2000). Predicting medication noncompliance after hospital discharge among patients with schizophrenia. Psychiatric Services, 51(2), 216-222.

25 Perese, Eris. F. (2007). Stigma, poverty, and victimization: Roadblocks to recovery for individuals with severe mental illness. Journal of the American Psychiatric Nurses Association, 13(5), 285-295.

26 Priebe, S., Watts, J., Chase, M., \& Matanov, A. (2005). Processes of disengagement and engagement in assertive outreach patients: Qualitative study. British Journal of Psychiatry, 187, 438-443.

27 Prince, J. D. (2005). Predicting outpatient mental health program withdrawal among recently discharged inpatients with schizophrenia. Psychological Services, 2(2), 142150.

28 Redlich, A. D., \& Monahan, J. (2006). General pressure to adhere to psychiatric treatment in the community. The International Journal of Forensic Mental Health, 5(2), 125-131.

29 Rohland, B. M., Rohrer, J. E., \& Richards, C. C. (2000). The long-term effects of outpatient commitment on service use. Administration and Policy in Mental Health, 27(6), 383394.

30 Roick, C., Heider, D., Kilian, R., Matschinger, H., Toumi, M., \& Angermeyer, M. C. (2004). Factors contributing to frequent use of psychiatric inpatient services by schizophrenia patients. Social Psychiatry and Psychiatric Epidemiology, 39(9), 744-751.

31 Rossi, A., Amaddeo, F., Bisoffi, G., Roggeri, M., Thornicroft, G., \& Tansella, M. (2002). Dropping out of care: Inappropriate terminations of contact with community-based psychiatric services. British Journal of Psychiatry, 181, 331-338.

32 Rossi, A., Ammaddeo, F., Sandri, M., Marsilio, A., Bianco, M., \& Tansella, M. (2008). What happens to patients only seen once by psychiatric services? Findings from a follow-up study. Psychiatry Research, 157(1-3), 53-65. 
33 Vogel, S., \& Hugelet, P. (1997). Factors associated with multiple admissions to a public psychiatric hospital. Acta Psychiatrica Scandinavica, 95(3), 244-253.

34 Vogt, D. (2011). Mental health-related beliefs as a barrier to service use for military personnel and veterans: A review. Psychiatric Services, 62(2), 135-142.

35 Walker, R., Minor-Schork, D., Bloch, R., \& Esinhart, J. (1996). High risk factors for rehospitalization within six months. Psychiatric Quarterly, 67(3), 235-243.

36 Webb, S., Yaguez, L., \& Langdon, P. E. (2007). Factors associated with multiple readmission to a psychiatric hospital. Journal of Mental Health, 16(5), 647-661.

37 Young, A. S., Grusky, O., Jordan, D., \& Belin, T. R. (2000). Routine outcome monitoring in a public mental health system: The impact of patients who leave care. Psychiatric Services, 51(1), 85-91. 